\title{
Circulating microRNA-196a is an early gastric cancer biomarker
}

\author{
Tsung-Hsing Chen ${ }^{1,2}{ }^{,}$Chieh Lee ${ }^{3}$, Cheng-Tang Chiu ${ }^{1}$, Yin-Yi Chu ${ }^{1}$, Hao-Tsai Cheng ${ }^{1}$, \\ Jun-Te Hsu ${ }^{4}$, Yung-Kuan Tsou ${ }^{1}$, Ren-Chin Wu ${ }^{5}$, Tse-Ching Chen ${ }^{5}$, Nien-Chen Chang ${ }^{6}$, \\ Ta-Sen Yeh ${ }^{4}$ and Kwang-Huei Lin ${ }^{6,7,8}$ \\ ${ }^{1}$ Department of Gastroenterology and Hepatology, Linkou Chang Gung Memorial Hospital, Chang Gung University College of \\ Medicine, Taoyuan, Taiwan \\ ${ }^{2}$ Graduate Institute of Clinical Medical Sciences, Chang Gung University, Taoyuan, Taiwan \\ ${ }^{3}$ Department of Industrial Engineering and Management, Yuan Ze University College of Engineering, Chung-Li City, Taiwan \\ ${ }^{4}$ Department of Surgery, Chang Gung Memorial Hospital, Linkou, Chang Gung University College of Medicine, Taoyuan, \\ Taiwan \\ ${ }^{5}$ Department of Pathology, Linkou Chang Gung Memorial Hospital, Chang Gung University College of Medicine, Taoyuan, \\ Taiwan \\ ${ }^{6}$ Graduate Institute of Biomedical Sciences, Chang Gung University, Taoyuan, Taiwan \\ ${ }^{7}$ Liver Research Center, Chang Gung Memorial Hospital, Linkou, Taoyuan, Taiwan \\ ${ }^{8}$ Research Center for Chinese Herbal Medicine, College of Human Ecology, Chang Gung University of Science and Technology \\ Taoyuan, Taiwan
}

Correspondence to: Kwang-Huei Lin, email: khlin@mail.cgu.edu.tw Ta-Sen Yeh, email: tsy471027@cgmh.org.tw

Keywords: microRNA-196a; precancerous gastric lesions; early gastric cancer; intestinal metaplasia; dysplasia

Received: April 11, 2017

Accepted: November 15, 2017

Published: December 07, 2017

Copyright: Chen et al. This is an open-access article distributed under the terms of the Creative Commons Attribution License 3.0 (CC BY 3.0), which permits unrestricted use, distribution, and reproduction in any medium, provided the original author and source are credited.

\section{ABSTRACT}

MicroRNA-196a (miRNA-196a) is associated with the development of gastric cancer and metastasis. Intestinal metaplasia and low- or high-grade dysplasia are considered to be precursors of intestinal type gastric cancer. Accordingly, we investigated the expression of plasma miRNA-196a as an early detection biomarker in precancerous gastric lesions and early cancer (pT1a/b), which is otherwise treated with endoscopic submucosal dissection. Our data showed that levels of circulating (plasma) miRNA-196a were higher in patients with precancerous lesions/early gastric adenocarcinoma than in healthy controls. The area under the receiver operating characteristic curve (AUC) for healthy controls vs. intestinal metaplasia was 0.9736; healthy controls vs. low-grade/high-grade dysplasia 0.9495; and healthy controls vs. early gastric cancer 0.9318 . These results indicate that circulating miRNA-196a is a novel biomarker for detection of early gastric cancer and its precursor.

\section{INTRODUCTION}

Gastric cancer (GC) remains a devastating disease, particularly in the advanced stage, due to the lack of biomarkers for early detection [1-3]. However, early intervention of precancerous gastric lesions and stage I GC leads to an excellent 5-year survival rate $\geq 95 \%$ [4]. Gastric cancer (GC) is histologically divided into two major types according to the Lauren classification: intestinal and diffuse. The former arises from atrophic gastritis (AG) and intestinal metaplasia (IM) [5-8]. IM is regarded as a "breaking point" [5-7] because H. pylori eradication results in histological improvement of gastric mucosa in AG (a precursor to IM) but not in IM. Currently, there is no clear consensus on gastric IM surveillance guidelines. However, early detection of GC may be achieved using an annual surveillance program if patients meet at least one of the following criteria: (1) IM extension $>20 \%$; (2) presence of incomplete type IM; (3) first-degree relative with gastric cancer; or (4) smokers [9].

Gastroscopy is a standard method for GC screening, but patients are reluctant to undergo this 
invasive procedure. It often fails to detect precancerous gastric lesions and early GC because superficial mucosal lesions are easily missed even by optimum preparation and advanced technology such as image enhancement endoscopy (IEE) $[10,11]$, and conventional computed tomography has a disappointing sensitivity rate $[12$, 13]. Currently, carcinoembryonic antigen (CEA) and carbohydrate antigen 19-9 (CA19-9) were reported with positive detection at levels as low as $20.9 \%$ and $34.6 \%$, respectively $[14,15]$. There remains an urgent need for serological biomarkers for precancerous gastric lesions and early gastric cancer detection.

Sun et al. [16], Li et al. [17], and our previous study [18] have shown that expression of miRNA-196a is implicated in the carcinogenesis of gastric cancer. We further demonstrated that circulating miRNA-196a is strongly associated with metastatic gastric cancer progression [19], suggesting that miRNA-196a acts as a circulating biomarker for gastric cancer. In this study, we measured the expression of circulating miRNA-196a in precancerous gastric lesions and early gastric cancer to determine if circulating miRNA-196a can be employed as a useful biomarker in clinical practice.

\section{RESULTS}

To verify circulating miRNA-196a expression as a biomarker of precancer and early gastric cancer, we recruited patients with intestinal metaplasia $(n=26)$, low/ high-grade dysplasia $(n=13)$ and early gastric cancer $(n=11)$ who had undergone gastroscopic biopsies with pathological proof; while another 32 healthy volunteers served as controls. The mean ages of healthy controls, intestinal metaplasia, low/high-grade dysplasia (LGD/ HGD), and early gastric cancer patients were $55 \pm 12,60$ $\pm 9,66 \pm 14$, and $64 \pm 13$, respectively. The expression of circulating miRNA-196a in health control, intestinal metaplasia, low/high - grade dysplasia and early gastric cancer detected by quantitative real-time polymerase chain reaction (qRT-PCR) were shown in Figure 1. It was found that expression of circulating miRNA-196a is lower in healthy control compared with all stages of pre-cancer $(p$ $<0.001, p<0.001, p<0.001)$ and early cancer $(p<0.01)$; whereas there was no difference among precancers and early gastric cancer $(p>0.05)$.

The AUC for healthy controls vs. IM was 0.9736 , the sensitivity and specificity were $96.15 \%$ and $87.50 \%$, respectively; for healthy controls vs. LGD/HGD, the AUC was 0.9495 , the sensitivity and specificity were $100.00 \%$ and $78.13 \%$; for healthy controls vs. early GC the AUC was 0.9318 , the sensitivity and specificity were $100.00 \%$ and $75.00 \%$ (Table 1 and Figure 2). The best cut-off points of miRNA-196a expression for each pre-cancer and early cancer stage are summarized as in Table 1. The logistic regression equations showed that the miRNA-196a is a significant predictor of whether a patient is healthy or at a specific pathological stage of the precancerous lesion (Table 2). To further demonstrate the effectiveness of miRNA-196a as a biomarker for GC, we implemented signal and detection theory to measure the distance between the signal and noise. The theory introduces a distance indicator discriminability index, d', which is calculated by hit (correct prediction), miss, false alarm, and correct rejection rates. A larger d' implies that the signal is further from the noise and suggests that miRNA-196a is a better pre-cancer or cancer indicator. The data for the hit, miss, false alarm and correct rejection rates of miRNA-196a expression in healthy volunteers vs. patients with pre-cancer and EC based on the cutoff points in Tables 1 and 2 are summarized in Table 3. The hit rate and the correct rejection rate are high. The miRNA-196a expression correctly identified all patients with LGD, HGD, and EC while keeping the false alarm rate relatively low. Similarly, miRNA-196a expression correctly identified over $96 \%$ of IM patients with a false alarm rate as low as $12.5 \%$. These results indicate that miRNA-196a is a very promising indicator of pre-cancer as well as cancer occurrence.

However, miRNA-196a expression did not significantly differ between pre-cancer patients and cancer patients. The AUC of IM vs. early GC was 0.7184 , and LGD/HGD vs. early GC was 0.7196 , both greater than 0.5 ( Figure 3 ). The d' of IM vs. early GC was 1.222 , and LGD/HGD vs. early GC was 4.0120 , both greater than 0 . We concluded that the miRNA-196a could weakly differentiate precancerous lesions from early cancer. Even though both the AUC and d' indicate that miRNA-196a could potentially serve as an indicator for pre-cancer or cancer, in practice, the hit rates were relatively low (Table 4).

\section{DISCUSSION}

Few studies have reported the detection of precancerous gastric lesions, particularly in the plasma. GC usually has a poor outcome as a result of the delay in diagnosis. Nevertheless, progression to cancer can be prevented if mucosal changes (e.g. AG or IM) are detected as early as possible. Endoscopic submucosal dissection can be done to excise the lesion once early gastric cancer or high-grade dysplasia is found while preserving the organ and promoting a good quality of life [20, 21]. At early stages most patients are asymptomatic, and the cancer is hard to detect, so it is challenging to prevent its progression. Although gastroscopy is a standard screening tool for gastric lesions, many patients consider it too uncomfortable. Thus, a liquid biopsy is more suitable for these asymptomatic patients, followed by gastroscopy to find those at risk.

The most pressing challenge is the identification of patients at high risk of diffuse or intestinal type GC. The latter is preceded by a sequence of precursor steps, which 
Table 1: Summary of cutoff points for different phases of diagnostics

\begin{tabular}{lcccccccc}
\hline & AUC & $\begin{array}{c}\text { Cutoff } \\
\text { Points }\end{array}$ & $\begin{array}{c}\mathbf{9 5 \%} \text { CI } \\
(\mathbf{L}, \mathbf{U})\end{array}$ & Sensitivity & $\begin{array}{c}\text { False } \\
\text { Negative }\end{array}$ & Specificity & $\begin{array}{c}\text { False } \\
\text { Positive }\end{array}$ & $\boldsymbol{P}$-Value \\
\hline H vs & & & & & & & & \\
IM+LGD/ & $96.55 \%$ & 36.2353 & $(35.282,37.189)$ & $74.36 \%$ & $25.64 \%$ & $96.88 \%$ & $3.12 \%$ & $<<0.05$ \\
HGD & & & & & & & & \\
H vs IM & $97.36 \%$ & 35.3679 & $(34.306,36.430)$ & $96.15 \%$ & $3.85 \%$ & $87.50 \%$ & $12.50 \%$ & $<<0.05$ \\
H vs LGD/ & $94.95 \%$ & 34.4784 & $(33.299,35.658)$ & $100.00 \%$ & $0.00 \%$ & $78.13 \%$ & $21.87 \%$ & $<<0.05$ \\
HGD & $93.18 \%$ & 32.7643 & $(31.568,33.961)$ & $100.00 \%$ & $0.00 \%$ & $75.00 \%$ & $25.00 \%$ & $<<0.05$ \\
H vs EC & &
\end{tabular}

Abbreviations: H: healthy control; IM: intestinal metaplasia; LGD: low-grade dysplasia; HGD: high-grade dysplasia; EC: early cancer; AUC: area under the curve.

Table 2: Summary of Logistics regression results for different phases of diagnostics

\begin{tabular}{lccccc}
\hline & Intercept & $\begin{array}{c}\mathbf{9 5 \%} \mathbf{C I} \\
(\mathbf{L}, \mathbf{U})\end{array}$ & $\begin{array}{c}\text { Estimated } \\
\text { Coefficient } \\
\text { of 49-eCT }\end{array}$ & $\begin{array}{c}\mathbf{9 5 \%} \mathbf{C I} \\
(\mathbf{L}, \mathbf{U})\end{array}$ & $\boldsymbol{P}$-Value \\
\hline H vs IM+LGD/ & -40.2805 & $(-63.763,-16.798)$ & 1.1519 & $(0.491,1.813)$ & $<0.05$ \\
HGD & -46.1239 & $(-77.975,-14.273)$ & 1.2992 & $(0.412,2.186)$ & $<0.05$ \\
H vs IM & -36.9854 & $(-64.962,-9.019)$ & 1.0332 & $(0.247,1.818)$ & $<0.05$ \\
H vs LGD/HGD & -25.1915 & $(-41.229,-9.154)$ & 0.7012 & $(0.247,1.156)$ & $<0.05$ \\
H vs EC &
\end{tabular}

Abbreviations: H: healthy control; IM: intestinal metaplasia; LGD: low-grade dysplasia; HGD: high-grade dysplasia.

Table 3: Summary of indicators from signal and detection theory for healthy control vs. patients in precancerous and early cancer stages

\begin{tabular}{|c|c|c|c|c|c|c|c|}
\hline Stages & Cutoff point & $\begin{array}{c}95 \% \text { CI } \\
(\mathrm{L}, \mathrm{U}) \\
\end{array}$ & Hit & $\begin{array}{c}\text { False } \\
\text { Alarm }\end{array}$ & Miss & Correct Rejection & $\mathbf{d}^{\prime}$ \\
\hline $\begin{array}{l}\mathrm{H} \text { vs. } \\
\mathrm{IM}+\mathrm{LGD} / \mathrm{HGD}\end{array}$ & 36.2353 & $(35.282,37.189)$ & $74.36 \%$ & $3.13 \%$ & $25.64 \%$ & $96.88 \%$ & 2.493 \\
\hline H vs IM & 35.3679 & $(34.306,36.430)$ & $96.15 \%$ & $12.50 \%$ & $3.85 \%$ & $87.50 \%$ & 2.919 \\
\hline $\begin{array}{l}\text { H vs LGD/ } \\
\text { HGD }\end{array}$ & 34.4784 & $(33.299,35.658)$ & $100.00 \%$ & $21.88 \%$ & $0.00 \%$ & $78.13 \%$ & 5.076 \\
\hline $\mathrm{H}$ vs EC & 32.7643 & $(31.568,33.961)$ & $100.00 \%$ & $25.00 \%$ & $0.00 \%$ & $75.00 \%$ & 4.974 \\
\hline
\end{tabular}

Abbreviations: H: healthy control; IM: intestinal metaplasia; LGD: low-grade dysplasia; HGD: high-grade dysplasia; EC: early cancer.

Table 4: Summary of indicators from signal and detection theory for precancerous and early cancer stages

\begin{tabular}{lccccccc}
\hline & $\begin{array}{c}\text { Cutoff } \\
\text { point }\end{array}$ & $\begin{array}{c}\mathbf{9 5 \%} \text { CI } \\
(\mathbf{L}, \mathbf{U})\end{array}$ & Hit & $\begin{array}{c}\text { False } \\
\text { Alarm }\end{array}$ & Miss & $\begin{array}{c}\text { Correct } \\
\text { Rejection }\end{array}$ & $\mathbf{d}^{\prime}$ \\
\hline IM vs LGD/HGD & 35.8114 & $(35.208,36.415)$ & $30.77 \%$ & $7.69 \%$ & $69.23 \%$ & $92.31 \%$ & 0.9240 \\
IM vs EC & 35.7565 & $(34.993,36.520)$ & $58.06 \%$ & $7.69 \%$ & $41.94 \%$ & $92.31 \%$ & 1.2220 \\
LGD/HGD vs EC & 34.2892 & $(33.344,35.234)$ & $38.71 \%$ & $0.00 \%$ & $61.29 \%$ & $100.00 \%$ & 4.0130 \\
\hline
\end{tabular}

Abbreviations: H: healthy control; IM: intestinal metaplasia; LGD: low-grade dysplasia; HGD: high-grade dysplasia; EC: early cancer. 
(colonic-type intestinal metaplasia) or small intestinal mucosa [22]. In our study, miRNA-196a expression is lower in healthy control compared with all stages of precancer, which might partially explain why IM is considered a "breaking point."

In our previous study, we found that circulating miRNA-196a/b could be used as a novel biomarker for metastatic gastric cancer prediction [18]. Here, we verified that the expression level of circulating miRNA-196a is a predictive biomarker for precancerous gastric lesions and GC. We found that miRNA-196a expression levels can distinguish healthy controls from those with precancerous gastric lesions (Table 1\&2 and Figure 1). The sensitivity and specificity of healthy controls vs. the IM+LGD/HGD group were $74.36 \%$ and $96.88 \%$; healthy controls vs. IM $96.15 \%$ and $87.50 \%$; healthy controls vs. LGD/HGD $100.00 \%$ and $78.13 \%$; healthy controls vs. EC $100.00 \%$ and $75.00 \%$. Our results show that circulating miRNA196a can distinguish patients with IM, LGD, HGD and early GC from healthy controls, and especially healthy participants from those subjects with gastric dysplasia and early cancer.

\section{MATERIALS AND METHODS}

\section{Subjects}

Plasma samples were collected from 50 patients (14 women and 36 men) from 2014 to 2016 at Chang Gung Memorial Hospital. The participants were divided into four subgroups, IM, low-grade dysplasia (LGD), highgrade dysplasia (HGD), and early gastric cancer (EC), and independently interpreted by two senior pathologists. A total of 32 healthy volunteers (18 women and 14 men) were enrolled from Chang Gung Memorial Hospital healthcare center and served as controls. These healthy volunteers were verified by negative results of routine blood test, chest radiographs, endoscopy examination (white light endoscopy), ultrasonography, cancer screening tests, and computed tomography /positron emission tomography scan. IEE was performed to validate patients with or without gastric mucosal change. The study was approved by the Institutional Review Board (IRB No. 103-2095C) of Chang Gung Memorial Hospital, and all participants gave informed consent.

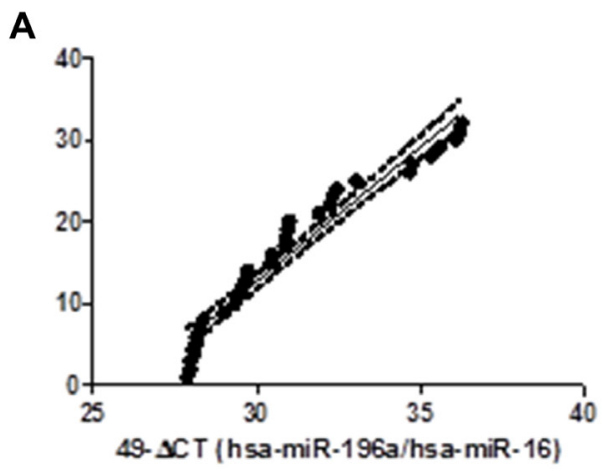

P-value $>0.05, \mathrm{HO}$ : Data is normally distributed.

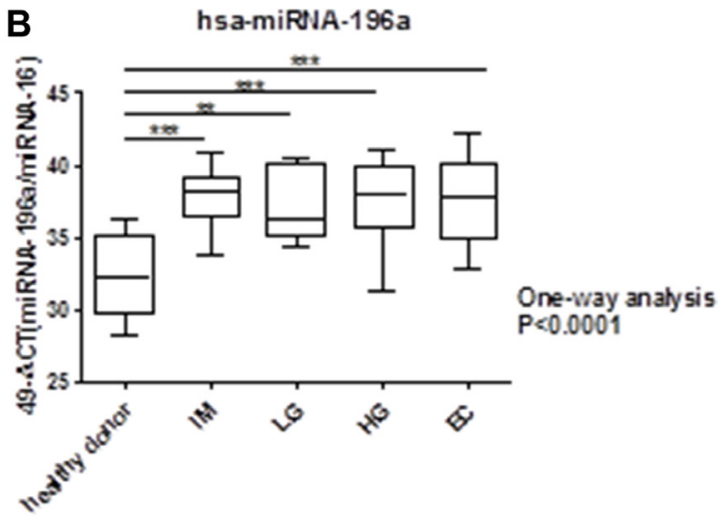

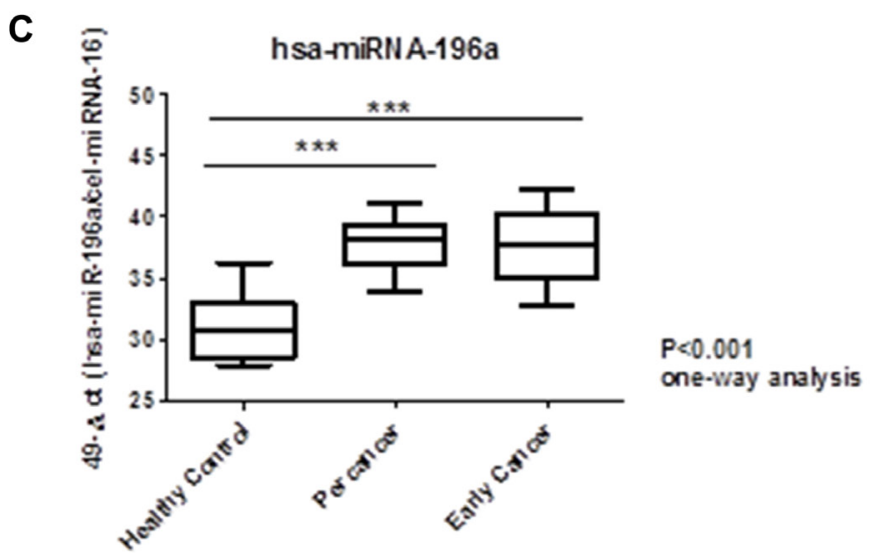

Figure 1: Normality test for healthy volunteers and boxplots of expression of miRNA196a in plasma. ${ }^{* * * *} P$-value $<0.001$ ${ }^{* *} \boldsymbol{P}$-value $<\mathbf{0 . 0 1}$. (A) Normality Test for Healthy Control. (B) Boxplots of Expression of miRNA-196a in Plasma for healthy donor (control), all stages of percancer and early cancer. (C) Boxplots of Expression of miRNA-196a in Plasma for healthy control, percancer, and early cancer. IM: intestinal metaplasia; LG: low-grade dysplasia; HG: high-grade dysplasia; EC: early cancer. 
A

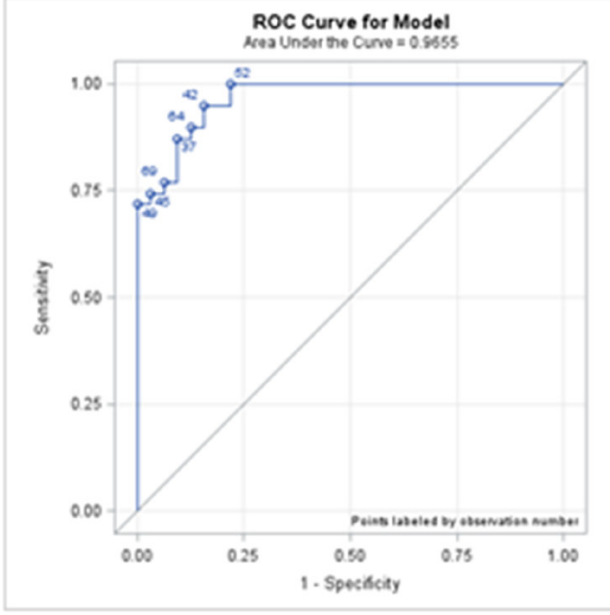

\section{C}

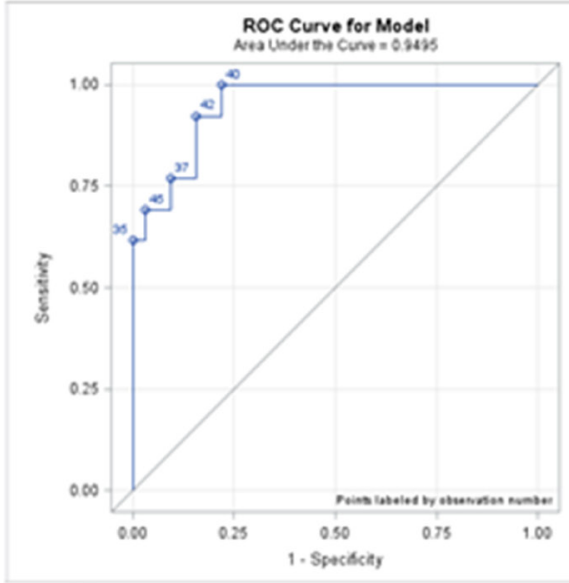

B

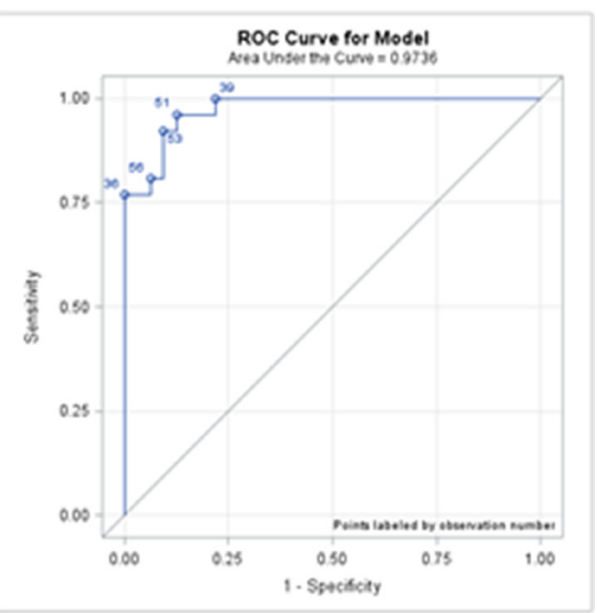

D

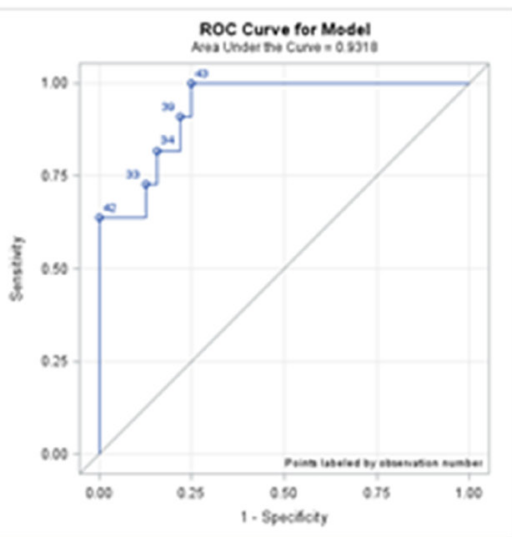

Figure 2: ROC curves for each phase. (A) ROC curve of healthy subjects vs. IM, HGD, and LGD; AUC was 0.9655. (B) ROC curve of healthy subjects vs. IM; AUC was 0.9736. (C) ROC curve of healthy subjects vs. LGD and HGD; AUC was 0.9495. (D) ROC curve of healthy subjects vs. EC; AUC was 0.9318. IM: intestinal metaplasia; LGD: low-grade dysplasia; HGD: high-grade dysplasia; EC: early cancer.

A

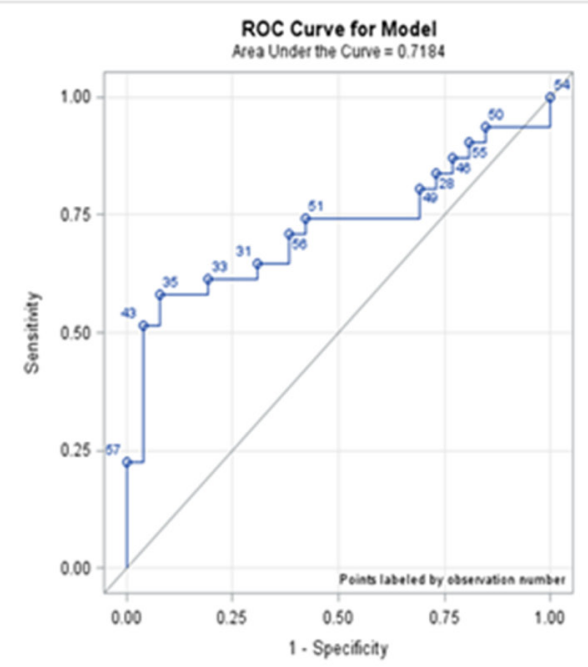

B

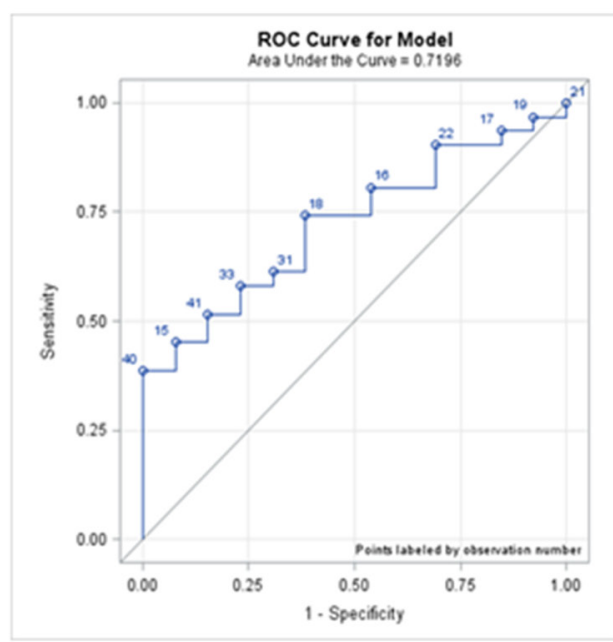

Figure 3: ROC curves for each phase. (A) ROC curve of IM vs. EC; AUC was 0.7184. (B) ROC curve of LGD/HGD, vs. EC; AUC was 0.7196 IM: intestinal metaplasia; LGD: low-grade dysplasia; HGD: high-grade dysplasia; EC: early cancer. 


\section{Quantification of plasma miRNA-196a}

The concentrations of miRNA from plasma samples were quantified using a NanoDrop 1000 spectrophotometer (Nanodrop Technologies Inc., Wilmington, DE, USA.). Circulating miRNA-196a expression was determined by quantitative real-time polymerase chain reaction (qRTPCR), as described previously, while miR-16 served as an internal control [18, 23-25]. Fluorescence emitted by SYBR green was assayed using the ABI Prism 7500 Fast Real-Time PCR system (Life Technologies). For plasma analysis, after normalization to the spike-in control (miR16), we calculated $49-\Delta \mathrm{Ct}$ to compare the values between patients and healthy control.

\section{Statistical analysis}

The $t$-test and a one-way analysis of variance (ANOVA) were used for pair-wise comparison of the plasma miRNA-196a expression in different stages of precancer and early cancer. The Kolmogorov-Smirnov test with $\alpha=0.05$ was used to verify the normality of healthy control samples. The receiver operating characteristic (ROC) curve and area under the curve (AUC) were used to assess the potential of using the plasma expression of miRNA-196a as a diagnostic tool for EC and precancerous lesions, such as incomplete and complete IM, LGD, and HGD. Youden's Index was employed to determine the theoretical best cut-off points for miRNA-196a expression between healthy volunteers, incomplete and complete IM (hence after IM), LGD, HGD, and EC. Signal detection theory was used to find the best practical cut-off points for miRNA-196a expression from IM to early cancer and LGD/HGD to early cancer. The AUC and d' were reported as indicators of the strength of the model. Signal and detection theory was implemented to determine the best practical cut-off points along with the probability of hit, miss, false alarm, and correct rejection. Descriptive data analyses were done using Prism 5 (GraphPad Software Inc., San Diego, CA, USA). Statistical analyses including ROC and signal detection theory were conducted using SAS 9.4 (SAS Institute Inc., Cary, NC, USA).

\section{CONCLUSIONS}

Circulating miRNA-196a is an effective biomarker that could afford patients with gastric dysplasia or early $\mathrm{GC}$ the chance to receive minimally invasive procedures to mitigate the disease progression. For example, we suggest that asymptomatic subjects with a high risk of developing gastric cancer could be screened by blood sampling for miRNA-196a. A follow-up with intensive gastroscopy (best combined with IEE) would be strongly advised for patients with significant changes in the circulating miRNA-196a level. Given that the number of patients with dysplasia and the total number of our study group was small, a large-scale study of circulating miRNA-196a as a biomarker for gastric pre-cancer detection is necessary.

\section{Author contributions}

Study concept and design; Tsung-Hsing Chen, ChengTang Chiu, Yin-Yi Chu, Hao-Tsai Cheng, Jun-Te Hsu, YungKuan Tsou, Ren-Chin Wu, Tse-Ching Chen, Nien-Chen Chang, Ta-Sen Yeh, Kwang-Huei Lin. Acquisition, analysis, and interpretation of data; Tsung-Hsing Chen, Nien-Chen Chang, Chieh Lee. Drafting of the manuscript: Tsung-Hsing Chen, Ta-Sen Yeh. Statistical analysis: Chieh Lee.

\section{ACKNOWLEDGMENTS AND FUNDING}

This work was supported by grants from ChangGung, Taoyuan, Taiwan (CMRPG3D1392).

\section{CONFLICTS OF INTEREST}

The authors declare no conflicting financial interests.

\section{REFERENCES}

1. Green D, Ponce de Leon S, Leon-Rodriguez E, SosaSanchez R. Adenocarcinoma of the stomach: univariate and multivariate analysis of factors associated with survival. Am J Clin Oncol. 2002; 25:84-89. https://doi. org/10.1097/00000421-200202000-00018.

2. Msika S, Benhamiche AM, Jouve JL, Rat P, Faivre J. Prognostic factors after curative resection for gastric cancer. A populationbased study. Eur J Cancer. 2000; 36:390-396. https://doi. org/10.1016/S0959-8049(99)00308-1.

3. Harrison LE, Karpeh MS, Brennan MF. Extended lymphadenectomy is associated with a survival benefit for node-negative gastric cancer. J Gastrointest Surg. 1998; 2:126-131. https://doi.org/10.1016/S1091255X(98)80002-4.

4. Craanen ME, Dekker W, Ferwerda J, Blok P, Tytgat GN. Early gastric cancer: a clinicopathologic study. J Clin Gastroenterol. 1991; 13:274-283. https://doi. org/10.1097/00004836-199106000-00006.

5. Correa P. Human gastric carcinogenesis: a multistep and multifactorial process-First American Cancer Society Award Lecture on Cancer Epidemiology and Prevention. Cancer Res. 1992; 52:6735-6740.

6. Busuttil RA, Boussioutas A. Intestinal metaplasia: a premalignant lesion involved in gastric carcinogenesis. J Gastroenterol Hepatol. 2009; 24:193-201. https://doi. org/10.1111/j.1440-1746.2008.05774.x.

7. Correa P, Piazuelo MB, Wilson KT. Pathology of gastric intestinal metaplasia: clinical implications. Am J Gastroenterol. 2010; 105:493-498. https://doi.org/10.1038/ ajg.2009.728. 
8. González CA, Pardo ML, Liso JM, Alonso P, Bonet C, Garcia RM, Sala N, Capella G, Sanz-Anquela JM. Gastric cancer occurrence in preneoplastic lesions: a long-term follow-up in a high-risk area in Spain. Int J Cancer. 2010; 127:2654-2660. https://doi.org/10.1002/ijc.25273.

9. Hirota WK, Zuckerman MJ, Adler DG, Davila RE, Egan J, Leighton JA, Qureshi WA, Rajan E, Fanelli R, WheelerHarbaugh J, Baron TH, Faigel DO, and Standards of Practice Committee, American Society for Gastrointestinal Endoscopy. ASGE guideline: the role of endoscopy in the surveillance of premalignant conditions of the upper GI tract. Gastrointest Endosc. 2006; 63:570-580. https://doi. org/10.1016/j.gie.2006.02.004.

10. Sugano K. Detection and management of early gastric cancer. Curr Treat Options Gastroenterol. 2015; 13:398408. https://doi.org/10.1007/s11938-015-0070-y.

11. Gotoda T, Shimoda T, Fujishiro M. Macroscopic feature of "gastritis-like cancer" with little malignant appearance in earlygastric cancer. [Article in Japanese] Stomach Intestine (Tokyo). 1999; 34:1495-503.

12. Hori S, Tsuda K, Murayama S, Matsushita M, Yukawa K, Kozuka T. CT of gastric carcinoma: preliminary results with a new scanning technique. Radiographics. 1992; 12:257268. https://doi.org/10.1148/radiographics.12.2.1561415.

13. Mani NB, Suri S, Gupta S, Wig JD. Two-phase dynamic contrast-enhanced computed tomography with waterfilling method for staging of gastric carcinoma. Clin Imaging. 2001; 25:38-43. https://doi.org/10.1016/S08997071(99)00134-5.

14. Leung WK, Wu MS, Kakugawa Y, Kim JJ, Yeoh KG, Goh KL, Wu KC, Wu DC, Sollano J, Kachintorn U, Gotoda T, Lin JT, You WC, et al, and Asia Pacific Working Group on Gastric Cancer. Screening for gastric cancer in Asia: current evidence and practice. Lancet Oncol. 2008; 9:279-287. https://doi.org/10.1016/S1470-2045(08)70072-X.

15. Marrelli D, Roviello F, De Stefano A, Farnetani M, Garosi L, Messano A, Pinto E. Prognostic significance of CEA, CA 19-9 and CA 72-4 preoperative serum levels in gastric carcinoma. Oncology. 1999; 57:55-62.

16. Sun M, Liu XH, Li JH, Yang JS, Zhang EB, Yin DD, Liu ZL, Zhou J, Ding Y, Li SQ, Wang ZX, Cao XF, De W. MiR$196 \mathrm{a}$ is upregulated in gastric cancer and promotes cell proliferation by downregulating p27(kip1). Mol Cancer Ther. 2012; 11:842-52.

17. Li HL, Xie SP, Yang YL, Cheng YX, Zhang Y, Wang J, Wang Y, Liu DL, Chen ZF, Zhou YN, Wu HY. Clinical significance of upregulation of mir-196a-5p in gastric cancer and enriched KEGG pathway analysis of target genes. Asian Pac J Cancer Prev. 2015; 16:1781-7.

18. Tsai MM, Wang CS, Tsai CY, Chen CY, Chi HC, Tseng YH, Chung PJ, Lin YH, Chung IH, Chen CY, Lin KH. MicroRNA-196a/-196b promote cell metastasis via negative regulation of radixin in human gastric cancer. Cancer Lett. 2014; 351:222-231. https://doi.org/10.1016/j. canlet.2014.06.004.

19. Tsai MM, Wang CS, Tsai CY, Huang CG, Lee KF, Huang HW, Lin YH, Chi HC, Kuo LM, Lu PH, Lin KH. Circulating microRNA-196a/b are novel biomarkers associated with metastatic gastric cancer. Eur J Cancer. 2016; 64:137-48.

20. Oka S, Tanaka S, Kaneko I, Mouri R, Hirata M, Kawamura T, Yoshihara M, Chayama K. Advantage of endoscopic submucosal dissection compared with EMR for early gastric cancer. Gastrointest Endosc. 2006 Dec; 64:877-83. https://doi.org/10.1016/j.gie.2006.03.932.

21. Gotoda T. Endoscopic resection of early gastric cancer: the Japanese perspective. Curr Opin Gastroenterol. 2006; 22:561569. https://doi.org/10.1097/01.mog.0000239873.06243.00.

22. Rugge M, Pennelli G, Pilozzi E, Fassan M, Ingravallo G, Russo VM, Di Mario F, and Gruppo Italiano Patologi Apparato Digerente (GIPAD), and Società Italiana di Anatomia Patologica e Citopatologia Diagnostica/ International Academy of Pathology, Italian division (SIAPEC/IAP). Gastritis: the histology report. Dig Liver Dis. 2011 (Suppl 4); 43:S373-384. https://doi.org/10.1016/ S1590-8658(11)60593-8.

23. Chen C, Ridzon DA, Broomer AJ, Zhou Z, Lee DH, Nguyen JT, Barbisin M, Xu NL, Mahuvakar VR, Andersen MR, Lao KQ, Livak KJ, Guegler KJ. Real-time quantification of microRNAs by stem-loop RT-PCR. Nucleic Acids Res. 2005; 33:e179. https://doi.org/10.1093/nar/gni178.

24. Shih $\mathrm{CH}$, Chen SL, Yen CC, Huang YH, Chen CD, Lee YS, Lin KH. Thyroid hormone receptor-dependent transcriptional regulation of fibrinogen and coagulation proteins. Endocrinology. 2004; 145:2804-2814. https://doi. org/10.1210/en.2003-1372.

25. Tsai MM, Lin PY, Cheng WL, Tsai CY, Chi HC, Chen CY, Tseng YH, Cheng YF, Chen CD, Liang Y, Liao CJ, Wu SM, Lin $\mathrm{YH}$, et al. Overexpression of ADP-ribosylation factor 1 in human gastric carcinoma and its clinicopathological significance. Cancer Sci 2012; 103:1136e44. 